\title{
Research on the BP-network-based Iris Recognition
}

\author{
Fengzhi Dai, Li Fan, Chunyu Yu, Bo Liu, Ruixiang Bai \\ College of Electronic Information and Automation, Tianjin University of Science and Technology, \\ 80 MailBox, 1038 Dagunanlu Road, Hexi District, Tianjin 300222, China \\ E-mail:daifz@tust.edu.cn \\ www.tust.edu.cn
}

\begin{abstract}
Iris recognition is the highly trusted identification recognition technology among methods of biological recognition. In this paper, we use the back propagation algorithm to train the neural network, so as to establish the iris recognition system model. The experiment demonstrates that it has a high recognition rate and the recognition speed is reasonable. The proposed method provides a convenient way for iris recognition.
\end{abstract}

Keywords: iris recognition, artificial neural network, back propagation algorithm, pattern recognition

\section{Introduction}

Iris is human's internal organization which is covered with a transparent membrane (cornea). It is hard to be forged. Also, likes the texture of fingerprint, the texture of iris is random determined in the period of gestation. The error probability of matching is quite low even though it is not technically testified that the iris's texture is absolutely unique ${ }^{1,2}$.

The iris is the colored, thin, circular structured muscle within the eye, which regulates the size of the pupil and thus controls the amount of light that enter the eye. Because the patterns of iris are formed randomly, even genetically identical twins will not have the same iris patterns $^{3}$. Also, iris is stable that it is immune to age and environment. Fig. 1 shows the iris and its position in the eye.

Many applications of iris recognition are used. The French ophthalmologist Alphonse Bertilon (1885) is considered to be the first for iris identification based on its color ${ }^{4}$. In 1985, ophthalmologists Leonard Flom and Aran Safir proposed that no two irises are exactly the same, and they got the patent application in $1987^{5}$. Also, Canada Border Services Agency introduces CANPass program ${ }^{6}$. Netherlands, Arab, Britain and Germany also

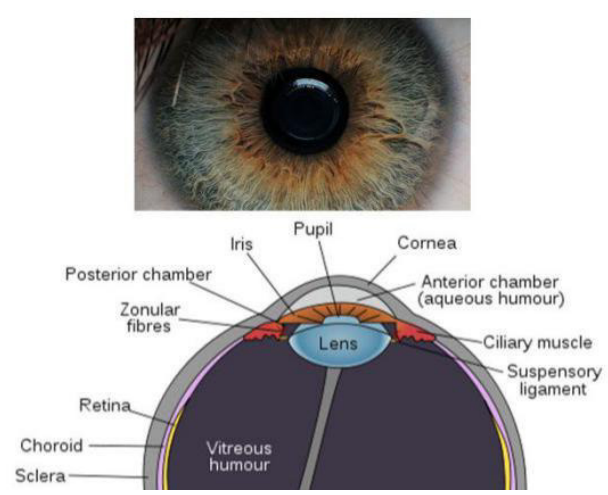

Fig. 1. Iris and its position in the eye

apply the iris recognition technology into immigration clearance. Meanwhile, many scientists have devoted to relevant study ${ }^{7}$. Boles and Boashash built a 1D representation of the gray level signature of the iris and applied it to zero-crossing of the dyadic wavelet ${ }^{8}$. Ma, Wang, and Tan used a bank of Gabor filters to capture the iris profile9. Poursaberi generated a binary code representation of the iris and used a minimum Euclidian distance for matching ${ }^{10}$. In 1995, the first commercial products became available ${ }^{11}$. 
In this paper, an algorithm that simplifies the procedure of iris recognition is proposed. It is characterized by: (1) Image pre-processing is used so as to avoid using complex mathematical algorithms. (2) The BP neural network is adopted. (3) Use gray-scale value of each pixel for learning and recognition.

\section{Neural Network}

Neuron is the basic unit of structure and function of the nervous system.

When a neuron is stimulated and excited, excitation will be converted into a signal and be conveyed to the synapse through axon. After the rapid diffusion of chemical molecules defused by the synapse in the synaptic space, the neighboring neurons generate new impulses and pass them to the next neurons in the same way.

This simplified mechanism of signal transfer constituted the fundamental step of early neurocomputing. In 1985, Rumelhart and McClelland research group proposed the multi-layer feed forward theory and the error back propagation (BP) algorithm base on the parallel distributed information processing. The BP neural network may contain one or several hidden layers (Fig.2).

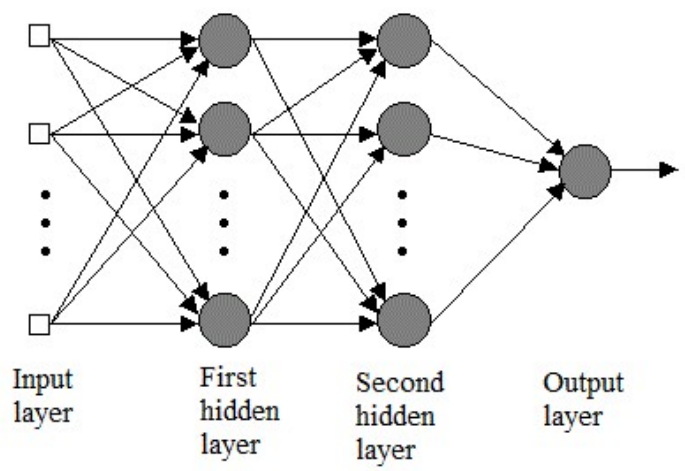

Fig. 2. Multi-layer neural network

\section{Iris Recognition}

\subsection{Image Pre-processing}

Colored iris images are selected to be pre-processed $^{12-14}$ to gray scale images (shown in Fig.3) and extracted the gray scale pixel features as the inputs to the neural network. All the iris images are processed by the OpenCV (the open source computer vision library), and converted the size to $100 \times 100$ pixels.

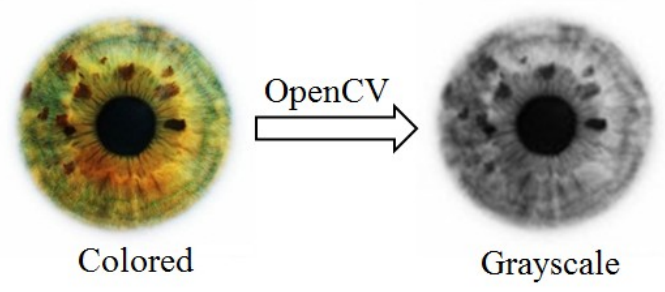

Fig. 3. Image processing

\subsection{Neural network design}

Firstly, the input and target samples of the neural network are obtained. We treat the pre-processed image as a $100 \times 100$ matrix, each element stores the gray scale pixel value as shown in Eq. (1). After that, the matrix is converted into a $10000 \times 1$ column vector that is treated as the input of the neural network (Fig.4), which has one hidden layer.

$\left[\begin{array}{cccccc}v 001001 & v 001002 & \ldots & v 001099 & v 001100 \\ v 002001 & v 002002 & & v 002099 & v 002100 \\ & \vdots & & & \vdots & \\ v 099001 & v 099002 & \ldots & v 099099 & v 099100 \\ v 100001 & v 100002 & & v 100099 & v 100100\end{array}\right]$

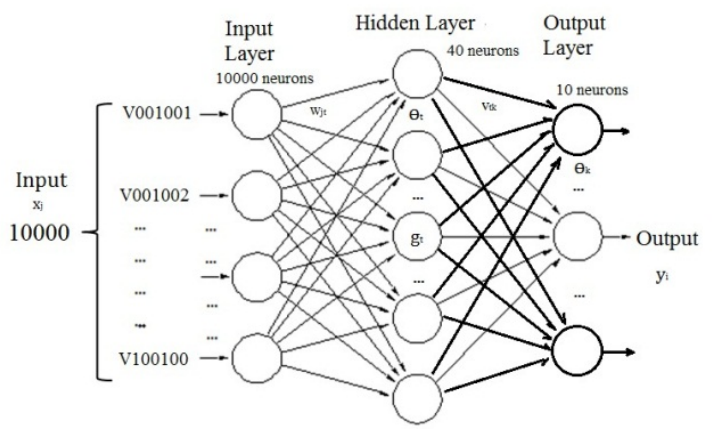

Fig. 4. The input of network

The input layer of the neural network has 10000 neurons. Learning procedure assumes that each input vector is paired with a target vector representing the desired output; together they are called a learning pair ${ }^{15}$. For the output layer, if 10 learning pairs are required, the output layer neurons are 10 . Output is restored in a $10 \times 1$ column vector.

For the number of hidden layer neurons, we refer to the empirical formula Eq.(2). We set the number of hidden layer neurons to 40 .

$$
n_{2} \leq \sqrt{n_{1}+n_{3}}+c
$$

Here, $n_{1}, n_{2}$ and $n_{3}$ are the numbers of the input, hidden and output layer neurons. $c$ is offset. 


\subsection{Neural network learning}

The learning rate $\eta$ and learning times are 0.4 and 5000 respectively. In Fig.4, all the weights $\left(W_{j t}, v_{t k}\right)$ and threshold values $\left(\theta_{t}, \theta_{k}\right)$ must be initialized to small random decimals before staring the learning process.

Training the BP neural network follows the five steps:

(1) Select the learning pair $\left(X^{p} Y^{p}\right.$ ) from the 10 pairs.

$$
\begin{aligned}
X^{p} & =\left(x_{1}, x_{2}, \ldots, x_{m}\right)^{\mathrm{T}} \\
Y^{p} & =\left(Y_{1}, Y_{2}, \ldots, Y_{m}\right)
\end{aligned}
$$

(2) Calculate the hidden and output vector of the neural network by Eq.(5) and Eq.(6) respectively.

$$
\begin{gathered}
g_{t}=f\left(\sum_{j=1}^{m} w_{j t} x_{j}-\theta_{j}\right)(t=1,2, \cdots, a) \\
\mathrm{y}_{\mathrm{k}}=\mathrm{f}\left(\sum_{\mathrm{t}=1}^{\mathrm{a}} \mathrm{v}_{\mathrm{tk}} \mathrm{g}_{\mathrm{t}}-\theta_{\mathrm{k}}\right)(\mathrm{k}=1,2, \cdots, \mathrm{n})
\end{gathered}
$$

(3) Calculate the general errors of each layer, $\delta_{k}^{p}$ and $\delta_{t}^{p}$.

(4) Adjust the weights of the network.

$$
\begin{aligned}
& v_{t k}(n+1)=v_{t k}(n)+\eta \cdot \sum_{p=1}^{P} \delta_{k}^{p} \cdot g_{t}^{p} \\
& w_{j t}(n+1)=w_{j t}(n)+\eta \cdot \sum_{p=1}^{p} \delta_{t}^{p} \cdot g_{j}^{p} \\
& \theta_{k}(n+1)=\theta_{k}(n)+\eta \cdot \sum_{p=1}^{p} \delta_{k}^{p} \\
& \theta_{t}(n+1)=\theta_{t}(n)+\eta \cdot \sum_{p=1}^{P} \delta_{t}^{p}
\end{aligned}
$$

(5) Repeat steps 1 through 4 for the 10 pairs until the sum squared error in Eq.(11) for the entire set is acceptable low.

$$
\mathrm{E}=\sum_{p=1}^{p} \sum_{k=1}^{n}\left(Y_{k}^{p}-y_{k}^{p}\right)^{2} / 2
$$

The result of the neural network learning is illustrated in Fig. 5. After 3000 times training, the sum squared error is acceptable low.

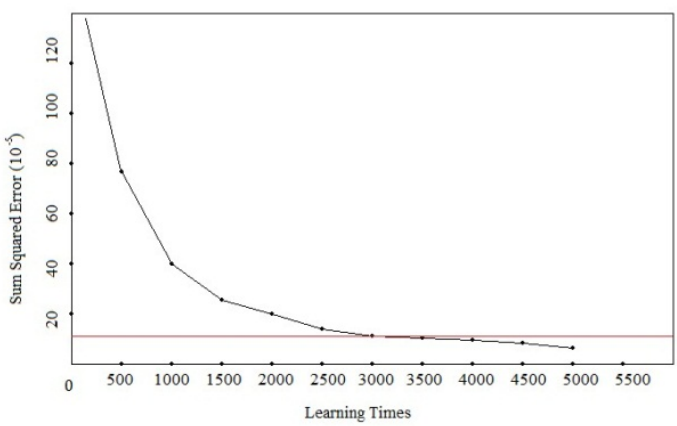

Fig. 5. Sum squared error for 5000 times learning

\subsection{Recognition and results}

The steps for recognition ${ }^{16}$ are shown in Fig.6: (1) Click the menu 'File' on the left top corner to load the original gray scale iris images. The 10 images are displayed.

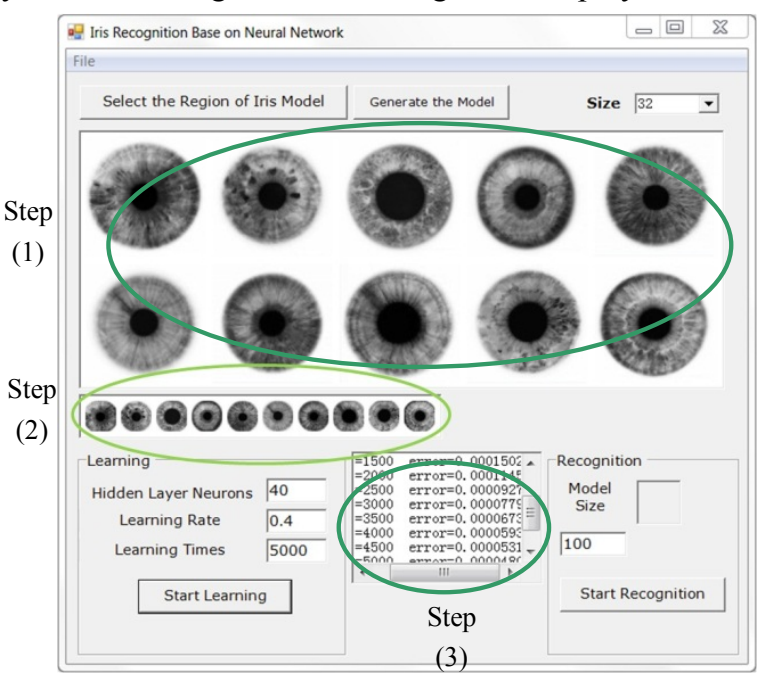

Fig.6. Neural network learning

(2) Select the region of iris model (as the $100 \times 100$ pixels) from the 10 iris images then generates the models. After that, 10 iris models will be converted to 10 matrices and displayed below the original images.

(3) Train the neural network by clicking the button "Start Learning", and the training procedure is also shown in Fig.6.

(4) Click the button "Start Recognition" to recognize whether the selected iris is one of those 10 irises or not.

The result of recognition is shown in Fig.7 and the recognized object is marked by the square. The experiment result shows the match rate is $99.946 \%$ to the selected object.

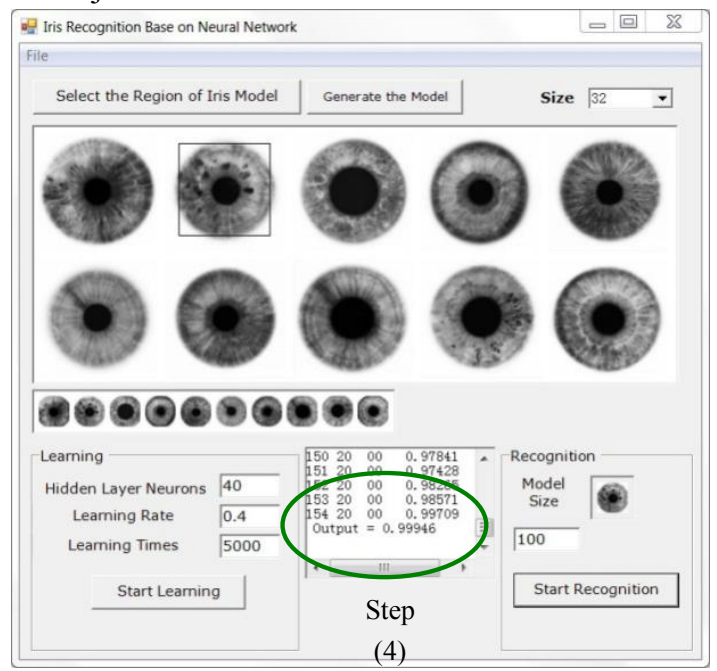

Fig.7. Recognition results 


\section{Conclusion}

In this paper, we design a neural network and used the back propagation algorithm to develop an elementary iris recognition system. The experiment demonstrates that the system has an ideal identification rate. With the development of the demand of identity recognition, this system can be applied in many fields such as security check and entrance guard.

In this study, the basic features of the iris is mainly extracted by image processing ${ }^{17}$, future research will use more shape and structural characteristics of the iris itself as the feature values.

\section{Acknowledgment}

The research is partly supported by the Scientific Research Foundation of Tianjin University of Science and Technology (20130123), and the Open Foundation (YF11700102) of Key Laboratory for Water Environment and Resources, Tianjin Normal University.

\section{References}

1. P. C. Kronfeld, The gross anatomy and embryology of the eye, The Eye, Vol. 1,(1968) pp. 1-66.

2. I. Mann, The Development of the Human Eye, (1950)

3. F. H. Adler, Physiology of the Eye, St. Louis, MO: Mosby (1965)

4. A.Bertillon, La couleur de l'iris, Revue scientifique, France, (1885)

5. L. Flom and A. Safir, Iris recognition system, U.S. Patent 4 $641349,1987$.
6. http://www.cbsa-asfc.gc.ca/media/facts-faits/009-eng.html, 04/07/2012

7. F.N. Sibai, Iris recognition using artificial neural networks, Expert Systems with Applications Vol.38, (2011) 59405946.

8. Boles. W. and Boashash. B., A human identification technique using images of the Iris and wavelet transform, IEEE Trans. Signal Processing, Vol. 46, No. 4 (1998) $1185-1188$.

9. Ma. L., Wang. Y. and Tan. T., Iris recognition using circular symmetric filters, In Proc. 16th Int. Conf. Pattern Recognition, Vol. 2, (2002) 414-417.

10. Poursaberi. A. and Araabi. B., A novel iris recognition system using morphological edge detector and wavelet phase features, ICGST-GVIP Journal, Vol. 5, No.6 (2005).

11. Irisian Technologies, Historical Timeline, (2003)

12. Yu C., Dai F., Zhang J., Design and application of iris recognition system based on neural network. WIT Trans. Inf. and Comm. Tech., Vol.46, (2013) pp.737-744.

13. Wildes. R. et al., A system for automated iris recognition, In Proc. 2nd IEEE workshop on applications of computer vision, pp.121-128

14. Lim, S., Lee, K., Byeon, O. and Kim, T., Efficient Iris recognition through improvement of feature vector and classifier, ETRI Journal, Vol.23, No.2, (2001) pp.1-2.

15. P. D. Wasserman, Neural computing: theory and practice, Van Nostrand Reinhold Co. New York, NY, USA (1989)

16. Sakai Kouichi, Foundation and Application of digital image processing by Visual C\#.NET and Visual Basic.NET (in Japanese), CQ Press (2006)

17. F. DAI, L. FAN, C. YU, B. LIU, Research on iris recognition based on the BP neural network. In Proc. 2015 Int. Conf. Artificial Life and Robotics, (2015) 178-182. 